\title{
Approximating Fixed Points of Operators Satisfying (RCSC) Condition in Banach Spaces
}

\author{
Thabet Abdeljawad $\mathbb{D},{ }^{1,2,3}$ Kifayat Ullah, ${ }^{4}$ Junaid Ahmad $\mathbb{D}^{,},{ }^{4}$ Manuel de la Sen $\mathbb{D}^{5},{ }^{5}$ \\ and Junaid Khan ${ }^{4}$ \\ ${ }^{1}$ Department of Mathematics and General Sciences, Prince Sultan University, P. O. Box 66833, Riyadh 11586, Saudi Arabia
${ }^{2}$ Department of Medical Research, China Medical University, Taichung 40402, Taiwan
${ }^{3}$ Department of Computer Sciences and Information Engineering, Asia University, Taichung, Taiwan
${ }^{4}$ Department of Mathematics, University of Science and Technology, Bannu, 28100 Khyber Pakhtunkhwa, Pakistan
${ }^{5}$ Institute of Research and Development of Processes, University of the Basque Country, Campus of Leioa (Bizkaia), P.O. Box \\ 644 Bilbao, Barrio Sarriena, 48940 Leioa, Spain
}

Correspondence should be addressed to Junaid Ahmad; ahmadjunaid436@gmail.com

Received 13 July 2020; Revised 25 August 2020; Accepted 2 September 2020; Published 22 September 2020

Academic Editor: Yuanfang Ru

Copyright (c) 2020 Thabet Abdeljawad et al. This is an open access article distributed under the Creative Commons Attribution License, which permits unrestricted use, distribution, and reproduction in any medium, provided the original work is properly cited.

\begin{abstract}
Let $K$ be a nonempty subset of a Banach space $E$. A mapping $T: K \rightarrow K$ is said to satisfy (RCSC) condition if each $a, b \in K$, $(1 / 2)\|a-F a\| \leq\|a-b\| \Rightarrow\|F a-F b\| \leq(1 / 3)(\|a-b\|+\|a-F b\|+\|b-F a\|)$. In this paper, we study, under some appropriate conditions, weak and strong convergence for this class of maps through $M$ iterates in uniformly convex Banach space. We also present a new example of mappings with condition (RCSC). We connect $M$ iteration and other well-known processes with this example to show the numerical efficiency of our results. The presented results improve and extend the corresponding results of the literature.
\end{abstract}

\section{Introduction}

$\mathbb{N}$ will denote the set of all natural numbers throughout. In 2008, Suzuki [1] introduced a new class of mappings as follow. A self-map $F$ on a subset $K$ of a Banach space $E$ is said to satisfy $(C)$ condition if for all $a, b \in K$, we have

$$
\frac{1}{2}\|a-F a\| \leq\|a-b\| \Rightarrow\|F a-F b\| \leq\|a-b\| .
$$

Obviously, when $F$ is nonexpansive mapping, that is, $\|$ $F a-F b\|\leq\| a-b \|$ holds for all $a, b \in K$, then $F$ satisfies the (C) condition. However, an example in [1] shows that there exists mappings, which satisfy the $(C)$ condition but not nonexpansive. A mapping with $(C)$ condition is often called Suzuki-type nonexpansive mapping. The class of Suzukitype nonexpansive mappings is extensively studied by many authors (cf. [2-12] and others).
In 2012, motivated by Suzuki $(C)$ condition, Karapinar [13] suggested a new condition on mappings, the so-called (RCSC) condition (or Reich-Chatterjea-Suzuki (C) condition). A self-map $F$ on a subset $K$ of a Banach space is said to satisfy the (RCSC) condition if for all $a, b \in K$, we have

$$
\begin{aligned}
\frac{1}{2}\|a-F a\| & \leq\|a-b\| \Rightarrow\|F a-F b\| \\
& \leq \frac{1}{3}(\|a-b\|+\|b-F a\|+\|a-F b\|) .
\end{aligned}
$$

The purpose of this work is to prove some weak and strong convergence results for this class of mappings through the $M$ iteration process [12] in the context of Banach spaces. We also give a numerical example to show the usefulness of our results. In this way, we extend and improve many wellknown corresponding results of the current literature. 
Approximating fixed points of nonlinear mappings played an important role and solved many problems [14-20]. It is now well known that if $F$ is nonexpansive, then the sequence of Picard iterates $w_{n+1}=F w_{n}$ may not converge to a fixed point of $F$. To overcome such problems and to get better a rate of convergence, many iterative processes are available in the literature. The wellknown iterative processes are the Mann [21], Ishikawa [22], Noor [23], Agarwal et al.S [24], Abbas and Nazir [25], Thakur et al. [7], Ullah and Arshad $M$ [12], and so on. Let $\alpha_{n}, \beta_{n}, \gamma_{n} \in(0,1), n \in \mathbb{N}$, and $F$ be a self-map on a nonempty convex subset $K$ of a Banach space.

The Mann iteration process [21] is a sequence $\left\{w_{n}\right\}$ defined as follows:

$$
\left.\begin{array}{l}
w_{1}=w \in K, \\
w_{n+1}=\left(1-\alpha_{n}\right) w_{n}+\alpha_{n} F w_{n} .
\end{array}\right\}
$$

The Ishikawa iteration process [22] is a sequence $\left\{w_{n}\right\}$ defined as follows:

$$
\left.\begin{array}{l}
w_{1}=w \in K, \\
s_{n}=\left(1-\beta_{n}\right) w_{n}+\beta_{n} F w_{n}, \\
w_{n+1}=\left(1-\alpha_{n}\right) w_{n}+\alpha_{n} F s_{n} .
\end{array}\right\}
$$

The Noor iteration process [23] is a sequence $\left\{w_{n}\right\}$ defined as follows:

$$
\left.\begin{array}{l}
w_{1}=w \in K, \\
v_{n}=\left(1-\gamma_{n}\right) w_{n}+\gamma_{n} F w_{n}, \\
s_{n}=\left(1-\beta_{n}\right) w_{n}+\beta_{n} F v_{n}, \\
w_{n+1}=\left(1-\alpha_{n}\right) w_{n}+\alpha_{n} F s_{n} .
\end{array}\right\}
$$
follows:

The $S$ iteration process [24] is a sequence $\left\{w_{n}\right\}$ defined as

$$
\left.\begin{array}{l}
w_{1}=w \in K, \\
s_{n}=\left(1-\beta_{n}\right) w_{n}+\beta_{n} F w_{n}, \\
w_{n+1}=\left(1-\alpha_{n}\right) F w_{n}+\alpha_{n} F s_{n} .
\end{array}\right\}
$$

The Abbas and Nazir iteration process [25] is a sequence $\left\{w_{n}\right\}$ defined as follows:

$$
\left.\begin{array}{l}
w_{1}=w \in K, \\
v_{n}=\left(1-\gamma_{n}\right) w_{n}+\gamma_{n} F w_{n}, \\
s_{n}=\left(1-\beta_{n}\right) F w_{n}+\beta_{n} F v_{n}, \\
w_{n+1}=\left(1-\alpha_{n}\right) F s_{n}+\alpha_{n} F v_{n} .
\end{array}\right\}
$$

The Thakur et al. iteration process [7] is a sequence $\left\{w_{n}\right\}$ defined as follows:

$$
\left.\begin{array}{l}
w_{1}=w \in K, \\
v_{n}=\left(1-\beta_{n}\right) w_{n}+\beta_{n} F w_{n}, \\
s_{n}=F\left(\left(1-\alpha_{n}\right) w_{n}+\alpha_{n} v_{n}\right), \\
w_{n+1}=F s_{n} .
\end{array}\right\}
$$

The $M$ iteration process [12] is a sequence $\left\{w_{n}\right\}$ defined as follows:

$$
\left.\begin{array}{l}
w_{1}=w \in K, \\
v_{n}=\left(1-\alpha_{n}\right) w_{n}+\alpha_{n} F w_{n}, \\
s_{n}=F v_{n}, \\
w_{n+1}=F s_{n} .
\end{array}\right\}
$$

In this paper, we will present some weak and strong convergence results using the $M$ iteration process (9) for mappings with (RCSC) condition. Similar results for the processes (3)-(8) can be proved on the same line of proofs.

\section{Preliminaries}

$p \in K$ is called a fixed point of a self-map $F$ on $K$ if $p=F p$. We will denote by $\operatorname{fix}(F)$ throughout the set of all fixed points of $F$. A Banach space $E$ is said to satisfy Opial condition [26] if and only if for each weakly convergent sequence $\left\{w_{n}\right\} \subseteq E$ with a weak limit $w \in E$, we have the following property:

$$
\liminf _{n \rightarrow \infty}\left\|w_{n}-w\right\|<\liminf _{n \rightarrow \infty}\left\|w_{n}-z\right\| \quad \text { for all } z \in E-\{w\} \text {. }
$$

A self-map $F$ on a subset $K$ of a Banach space is said to satisfy the condition $I$ [27] if there is nondecreasing function $g:[0, \infty) \longrightarrow[0, \infty)$ with the properties $g(0)=0, g(z)>0$ for every $z>0$, and $\|a-F a\| \geq g(\operatorname{dist}(a, \operatorname{fix}(F)))$ for all $a \in K$.

Let $K$ be a nonempty subset of a Banach space $E$ and $\left\{w_{n}\right\}$ a bounded sequence in $E$. For each $x \in E$, define

(i) asymptotic radius of $\left\{w_{n}\right\}$ at $x$ by $r\left(x,\left\{w_{n}\right\}\right):=$ $\limsup _{n \rightarrow \infty}\left\|x-w_{n}\right\|$

(ii) asymptotic radius of $\left\{w_{n}\right\}$ relative to $K$ by $r(K$, $\left.\left\{w_{n}\right\}\right)=\inf \left\{r\left(x,\left\{w_{n}\right\}\right): x \in K\right\}$

(iii) asymptotic center of $\left\{w_{n}\right\}$ relative to $K$ by $A(K$, $\left.\left\{w_{n}\right\}\right)=\left\{x \in K: r\left(x,\left\{w_{n}\right\}\right)=r\left(K,\left\{w_{n}\right\}\right)\right\}$

When the space $E$ is uniformly convex [28], then the set $A\left(K,\left\{w_{n}\right\}\right)$ is always singleton. Notice also that the set $A\left(K,\left\{w_{n}\right\}\right)$ is convex as well as nonempty provided that $K$ is weakly compact convex (see, e.g., $[29,30]$ ).

Lemma 1. [13]. 
Let $F$ be a self-map on a subset $K$ of a Banach space. If $F$ satisfies the (RCSC) condition, then for all $a, b \in K$, the following holds:

$$
\|a-F b\| \leq 9\|a-F a\|+\|a-b\| .
$$

The following facts are also needed.

Lemma 2. [13].

Let $E$ be a Banach space having Opial's property, $\varnothing \neq K$ $\subseteq E$ and $F: K \longrightarrow K$. If $F$ satisfies the condition (RCSC), then the following condition holds:

$$
\left\{w_{n}\right\} \subseteq K, w_{n} \rightarrow w,\left\|w_{n}-F w_{n}\right\| \longrightarrow 0 \Rightarrow F w=w .
$$

The following lemma gives the structure of the fixed point set associated with a mapping satisfying (RCSC) condition.

\section{Lemma 3. [13].}

Let $F$ be a self-map on a subset $\varnothing \neq K$ of a Banach space. If $F$ satisfies the (RCSC) condition, then fix $(F)$ is closed. Moreover, if $E$ is strictly convex and $K$ is convex, then fix $(F)$ is also convex.

\section{Lemma 4. [13].}

Let $F$ be a self-map on a subset $\varnothing \neq K$ of a Banach space. If $F$ satisfies (RCSC) condition, then for all $a \in K$ and $p \in f i x(F)$, $\|F a-F p\| \leq\|a-p\|$ holds.

\section{Lemma 5. [31].}

Let $0<x \leq \eta_{n} \leq y<1$ for each $n \in \mathbb{N}$ and $\left\{v_{n}\right\}$ and $\left\{w_{n}\right\}$ be any two sequences in a uniformly convex Banach space $E$ such that $\limsup _{n \rightarrow \infty}\left\|v_{n}\right\| \leq \zeta$, $\limsup _{n \rightarrow \infty}\left\|w_{n}\right\| \leq \zeta$, and $\lim _{n \rightarrow \infty}\left\|\eta_{n} v_{n}+\left(1-\eta_{n}\right) w_{n}\right\|=\zeta$ for some $\zeta \geq 0$; then, $\lim _{n \rightarrow \infty}\left\|v_{n}-w_{n}\right\|=0$.

\section{Main Results}

We begin this section by proving a crucial lemma.

Lemma 6. Let $F$ be a self-map on a subset $\varnothing \neq K$ of a Banach space. Assume that F satisfies the (RCSC) condition and let $\left\{w_{n}\right\}$ be a sequence generated by (9). If $f i x(F) \neq \varnothing$, then $\lim _{n \rightarrow \infty}\left\|w_{n}-p\right\|$ exists for each $p \in \operatorname{fix}(F)$.

Proof. Let $p \in \operatorname{fix}(F)$ and $n \in \mathbb{N}$. By Lemma 4, we have

$$
\begin{aligned}
\left\|v_{n}-p\right\| & =\left\|\left(1-\alpha_{n}\right) w_{n}+\alpha_{n} F w_{n}-p\right\| \\
& \leq\left(1-\alpha_{n}\right)\left\|w_{n}-p\right\|+\alpha_{n}\left\|F w_{n}-p\right\| \\
& \leq\left(1-\alpha_{n}\right)\left\|w_{n}-p\right\|+\alpha_{n}\left\|w_{n}-p\right\|=\left\|w_{n}-p\right\|
\end{aligned}
$$

which implies that

$$
\begin{aligned}
\left\|w_{n+1}-p\right\| & =\left\|s_{n}-p\right\| \leq\left\|s_{n}-p\right\|=\left\|F v_{n}-p\right\| \\
& \leq\left\|v_{n}-p\right\| \leq\left\|w_{n}-p\right\| .
\end{aligned}
$$

Hence, $\left\|w_{n+1}-p\right\| \leq\left\|w_{n}-p\right\|$ for all $n \in \mathbb{N}$ and $p \in \operatorname{fix}(F)$. Thus, $\left\{\left\|w_{n}-p\right\|\right\}$ is bounded and nonincreasing, which implies that $\lim _{n \rightarrow \infty}\left\|w_{n}-p\right\|$ exists for each $p \in \operatorname{fix}(F)$.

Now we give the necessary and sufficient condition for the existence of a fixed point for mapping with (RCSC) condition defined on a nonempty closed convex subset of a complete uniformly convex Banach space.

Theorem 7. Let $F$ be a self-map on a closed convex subset $\varnothing$ $\neq K$ of a uniformly convex Banach space. Assume that $F$ satisfies the (RCSC) condition and let $\left\{w_{n}\right\}$ be a sequence generated by (9). Then, $f i x(F) \neq \varnothing$ if and only if $\left\{w_{n}\right\}$ is bounded and $\lim _{n \rightarrow \infty}\left\|F w_{n}-w_{n}\right\|=0$.

Proof. Let $\left\{w_{n}\right\}$ be bounded and $\lim _{n \rightarrow \infty}\left\|F w_{n}-w_{n}\right\|=0$. Let $p \in A\left(K,\left\{w_{n}\right\}\right)$. By Lemma 1 , we have

$$
\begin{aligned}
& r\left(F p,\left\{w_{n}\right\}\right)=\limsup _{n \rightarrow \infty}\left\|w_{n}-F p\right\| \\
& \leq \underset{n \rightarrow \infty}{\operatorname{9limsup}}\left\|F w_{n}-w_{n}\right\|+\underset{n \rightarrow \infty}{\limsup }\left\|w_{n}-p\right\| \\
& =\underset{n \rightarrow \infty}{\limsup }\left\|w_{n}-p\right\|=r\left(p,\left\{w_{n}\right\}\right) .
\end{aligned}
$$

Hence, we conclude that $F p \in A\left(K,\left\{w_{n}\right\}\right.$. Since $E$ is uniformly convex, $A\left(K,\left\{w_{n}\right\}\right)$ consists of a unique element. Thus, we have $F p=p$.

Conversely, suppose that $\operatorname{fix}(F) \neq \varnothing$ and $p \in \operatorname{fix}(F)$. By Lemma $6, \lim _{n \rightarrow \infty}\left\|w_{n}-p\right\|$ exists and $\left\{w_{n}\right\}$ is bounded. Put

$$
\lim _{n \rightarrow \infty}\left\|w_{n}-p\right\|=\zeta
$$

From (13), we have

$$
\begin{aligned}
\left\|v_{n}-p\right\| & \leq\left\|w_{n}-p\right\| \Rightarrow \underset{n \rightarrow \infty}{\limsup }\left\|v_{n}-p\right\| \\
& \leq \limsup _{n \rightarrow \infty}\left\|w_{n}-p\right\|=\zeta .
\end{aligned}
$$

By Lemma 4, we have

$$
\begin{aligned}
\left\|F w_{n}-p\right\| & \leq\left\|w_{n}-p\right\| \Rightarrow \underset{n \rightarrow \infty}{\limsup }\left\|F w_{n}-p\right\| \\
& \leq \limsup _{n \rightarrow \infty}\left\|w_{n}-p\right\|=\zeta .
\end{aligned}
$$

From (14), we have

$$
\begin{aligned}
\left\|w_{n+1}-p\right\| & \leq\left\|v_{n}-p\right\| \Rightarrow \zeta=\liminf _{n \rightarrow \infty}\left\|w_{n+1}-p\right\| \\
& \leq \liminf _{n \rightarrow \infty}\left\|v_{n}-p\right\| .
\end{aligned}
$$

From (17) and (19), we have

$$
\zeta=\lim _{n \rightarrow \infty}\left\|v_{n}-p\right\| .
$$


From (20), we have

$$
\zeta=\lim _{n \rightarrow \infty}\left\|v_{n}-p\right\|=\lim _{n \rightarrow \infty}\left\|\left(1-\alpha_{n}\right)\left(w_{n}-p\right)+\alpha_{n}\left(F w_{n}-p\right)\right\|
$$

Hence,

$$
\zeta=\lim _{n \rightarrow \infty}\left\|\left(1-\alpha_{n}\right)\left(w_{n}-p\right)+\alpha_{n}\left(F w_{n}-p\right)\right\|
$$

By Lemma 5, we have

$$
\lim _{n \rightarrow \infty}\left\|F w_{n}-w_{n}\right\|=0
$$

Now we can prove the following weak convergence theorem.

Theorem 8. Let $F$ be a self-map on a closed convex subset $\varnothing$ $\neq K$ of a uniformly convex Banach space $E$ having Opial's property. Assume that $F$ satisfies the (RCSC) condition with fix $(F) \neq \varnothing$ and let $\left\{w_{n}\right\}$ be a sequence generated by (9). Then, $\left\{w_{n}\right\}$ converges weakly to a fixed point of $F$.

Proof. Since $E$ is uniformly convex, $E$ is reflexive. By Theorem $7,\left\{w_{n}\right\}$ is bounded and $\lim _{n \rightarrow \infty}\left\|F w_{n}-w_{n}\right\|=0$ for all $n \in \mathbb{N}$. By the reflexivity, one can find a weakly convergent subsequence $\left\{w_{n_{i}}\right\}$ of $\left\{w_{n}\right\}$ with a weak limit say $w \in K$. By Lemma 2, we have $F w=w$. It is suffice to show that $w$ is the weak limit of $\left\{w_{n}\right\} . w$ is not the weak limit of $\left\{w_{n}\right\}$. Then, one can find another weakly convergent subsequence $\left\{w_{n_{j}}\right\}$ of $\left\{w_{n}\right\}$ with a weak limit $w^{\prime}$ such that $w^{\prime} \neq w$. Again by Lemma 2, $F w^{\prime}=w^{\prime}$. By Lemma 6 together with Opial's property, we have

$$
\begin{aligned}
\lim _{n \rightarrow \infty}\left\|w_{n}-w\right\| & =\lim _{i \rightarrow \infty}\left\|w_{n_{i}}-w\right\|<\lim _{i \rightarrow \infty}\left\|w_{n_{i}}-w^{\prime}\right\| \\
& =\lim _{n \rightarrow \infty}\left\|w_{n}-w^{\prime}\right\|=\lim _{j \rightarrow \infty}\left\|w_{n_{j}}-w^{\prime}\right\| \\
& <\lim _{j \rightarrow \infty}\left\|w_{n_{j}}-w\right\|=\lim _{n \rightarrow \infty}\left\|w_{n}-w\right\| .
\end{aligned}
$$

This is a contradiction. So, we have $w=w^{\prime}$. Hence, $w$ is the weak limit of $\left\{w_{n}\right\}$

Now we prove a strong convergence theorem as follows.

Theorem 9. Let $F$ be a self-map on a compact convex subset $\varnothing \neq K$ of a uniformly convex Banach space. Assume that $F$ satisfies the (RCSC) condition with fix $(F) \neq \varnothing$ and let $\left\{w_{n}\right\}$ be a sequence generated by (9). Then, $\left\{w_{n}\right\}$ converges strongly to a fixed point of $F$.

Proof. By Theorem $7, \lim _{n \rightarrow \infty}\left\|F w_{n}-w_{n}\right\|=0$ for all $n \in \mathbb{N}$. Since $K$ is compact and convex, we can find a strongly convergent subsequence $\left\{w_{n_{j}}\right\}$ of $\left\{w_{n}\right\}$ with a strong limit say q. By Lemma 1 , we have

$$
\left\|w_{n_{j}}-F q\right\| \leq 9\left\|w_{n_{j}}-F w_{n_{j}}\right\|+\left\|w_{n_{j}}-q\right\| \longrightarrow 0
$$

By the uniqueness of limits in Banach spaces, $F q=q$. By Lemma $6, \lim _{n \rightarrow \infty}\left\|w_{n}-q\right\|$ exists and hence $q$ is the strong limit of $\left\{w_{n}\right\}$.

Now we state the following theorem. Since the proof is elementary, we will not include the details.

Theorem 10. Let $F$ be a self-map on a closed convex subset $\varnothing \neq K$ of a uniformly convex Banach space. Assume that $F$ satisfies the (RCSC) condition with fix $(F) \neq \varnothing$ and let $\left\{w_{n}\right\}$ be a sequence generated by (9). Then, $\left\{w_{n}\right\}$ converges strongly to a fixed point of $F$ provided that liminf ${ }_{n \rightarrow \infty}$ dist $\left(w_{n}, f i x(F)\right)=0$.

The following convergence theorem is based on condition $I$.

Theorem 11. Let $F$ be a self-map on a closed convex subset $\varnothing \neq K$ of a uniformly convex Banach space. Assume that $F$ satisfies the (RCSC) condition with fix $(F) \neq \varnothing$ and let $\left\{w_{n}\right\}$ be a sequence generated by (9). Then, $\left\{w_{n}\right\}$ converges strongly to a fixed point of $F$ provided that $F$ satisifes the condition $I$.

Proof. By Theorem 7, it follows that $\liminf _{n \rightarrow \infty}\left\|w_{n}-F w_{n}\right\|$ $=0$. By the condition $I$, we have $\liminf _{n \rightarrow \infty} \operatorname{dist}\left(w_{n}, \operatorname{fix}(F)\right)$ $=0$. The conclusion follows from Theorem 10 .

\section{Example}

In this section, we compare the rate of convergence of the $M$ iteration process with other iterations in the setting of mappings with (RCSC) condition.

Example 1. Let $K=[2,5]$ be endowed with the usual norm. Set $F a=2$ if $a=5$ and $F a=(2+a) / 2$ if $a \neq 5$. We shall prove that $F$ satisifes the (RCSC) condition. The case when $a, b \in\{5\}$ is trivial. We consider only the following three nontrivial cases.

When $a, b \in[2,5)$, then $F a=(2+a) / 2$ and $F b=(2+b) / 2$. Using triangle inequality, we have

$$
\begin{aligned}
|F a-F b| & =\frac{1}{2}|a-b| \leq \frac{1}{3}|a-b|+\frac{1}{2}|a-b| \\
& =\frac{1}{3}|a-b|+\frac{1}{3}\left|\frac{3 a}{2}-\frac{3 b}{2}\right| \\
& =\frac{1}{3}|a-b|+\frac{1}{3}\left|\left(a-\left(\frac{2+b}{2}\right)\right)-\left(b-\left(\frac{2+a}{2}\right)\right)\right| \\
& \leq \frac{1}{3}|a-b|+\frac{1}{3}\left|a-\left(\frac{2+b}{2}\right)\right|+\frac{1}{3}\left|b-\left(\frac{2+a}{2}\right)\right| \\
& =\frac{1}{3}(|a-b|+|a-F b|+|b-F a|) .
\end{aligned}
$$


TABle 1: Computation table obtained from the $M$, Thakur et al., Abbas and Nazir, S, Noor, Ishikawa, and Mann iterates for mapping $F$ defined in Example 1.

\begin{tabular}{|c|c|c|c|c|c|c|c|}
\hline$n$ & $M$ & $\begin{array}{c}\text { Thakur } \\
\text { et al. }\end{array}$ & $\begin{array}{c}\text { Abbas } \\
\text { and } \\
\text { Nazir }\end{array}$ & $S$ & Noor & Ishikawa & Mann \\
\hline 1 & 3 & 3 & 3 & 3 & 3 & 3 & 3 \\
\hline 2 & 2.1625 & 2.1931 & 2.2456 & 2.3863 & 2.4851 & 2.5363 & 2.6500 \\
\hline 3 & 2.0264 & 2.0373 & 2.0603 & 2.1492 & 2.2353 & 2.2876 & 2.4225 \\
\hline 4 & 2.0043 & 2.0072 & 2.0148 & 2.0576 & 2.1141 & 2.1542 & 2.2746 \\
\hline 5 & 2.0007 & 2.0014 & 2.0036 & 2.0223 & 2.0554 & 2.0827 & 2.1785 \\
\hline 6 & 2.0001 & 2.0003 & 2.0009 & 2.0086 & 2.0269 & 2.0443 & 2.1160 \\
\hline 7 & 2 & 2.0001 & 2.0002 & 2.0033 & 2.0130 & 2.0238 & 2.0754 \\
\hline 8 & 2 & 2 & 2.0001 & 2.0013 & 2.0063 & 2.0123 & 2.0490 \\
\hline 9 & 2 & 2 & 2 & 2.0005 & 2.0031 & 2.0068 & 2.0318 \\
\hline 10 & 2 & 2 & 2 & 2.0002 & 2.0015 & 2.0037 & 2.0207 \\
\hline 11 & 2 & 2 & 2 & 2.0001 & 2.0007 & 2.0020 & 2.0134 \\
\hline 12 & 2 & 2 & 2 & 2 & 2.0003 & 2.0011 & 2.0088 \\
\hline 13 & 2 & 2 & 2 & 2 & 2.0002 & 2.0006 & 2.0057 \\
\hline 14 & 2 & 2 & 2 & 2 & 2.0001 & 2.0003 & 2.0037 \\
\hline 15 & 2 & 2 & 2 & 2 & 2 & 2.0002 & 2.0024 \\
\hline 16 & 2 & 2 & 2 & 2 & 2 & 2.0001 & 2.0016 \\
\hline 17 & 2 & 2 & 2 & 2 & 2 & 2 & 2.0010 \\
\hline 18 & 2 & 2 & 2 & 2 & 2 & 2 & 2.0007 \\
\hline 19 & 2 & 2 & 2 & 2 & 2 & 2 & 2.0004 \\
\hline 20 & 2 & 2 & 2 & 2 & 2 & 2 & 2.0003 \\
\hline 21 & 2 & 2 & 2 & 2 & 2 & 2 & 2.0002 \\
\hline 22 & 2 & 2 & 2 & 2 & 2 & 2 & 2.0001 \\
\hline 23 & 2 & 2 & 2 & 2 & 2 & 2 & 2 \\
\hline
\end{tabular}

When $a \in[2,5)$ and $b \in\{5\}$, then $F a=(2+a) / 2$ and $F b=2$. Now

$$
\begin{aligned}
|F a-F b| & =\left|\left(\frac{a-2}{2}\right)-2\right|=\left|\frac{a-2}{2}\right|=\frac{1}{3}\left(\left|\frac{3 a-6}{2}\right|\right) \\
& =\frac{1}{3}\left(\left|\frac{a-2}{2}+(a-2)\right|\right) \leq \frac{1}{3}\left|\frac{a-2}{2}\right|+\frac{1}{3}|a-2| \\
& =\frac{1}{3}\left|(a-b)+\left(b-\left(\frac{2+a}{2}\right)\right)\right|+\frac{1}{3}|a-2| \\
& \leq \frac{1}{3}|a-b|+\frac{1}{3}\left|b-\left(\frac{2+a}{2}\right)\right|+\frac{1}{3}|a-2| \\
& =\frac{1}{3}(|a-b|+|a-F b|+|b-F a|) .
\end{aligned}
$$

Finally, when $a \in\{5\}$ and $b \in[2,5)$, then $F a=2$ and $F b=(2+b) / 2$. Now

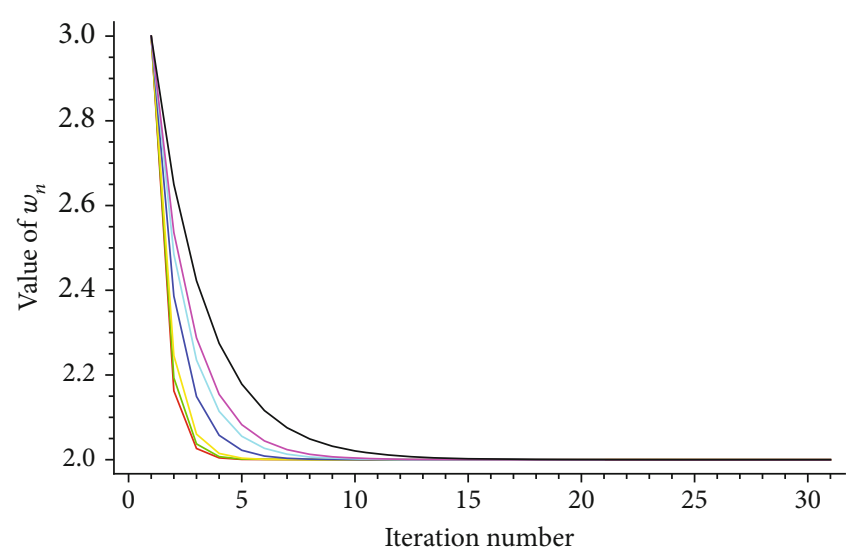

Figure 1: Convergence behavior of the $M$ (red line), Thakur et al. (green line), Abbas and Nazir (yellow line), Agarwal et al. (blue line), Noor (cyan line), Ishikawa (magenta line), and Mann (black line) iterates for mapping $F$ defined in Example 1 where $w_{1}=3$.

TABLE 2: $\quad \alpha_{n}=2 n / \sqrt{7 n+11}$ and $\beta_{n}=1 / \sqrt{3 n+9}$. Number of iterations required to obtain the fixed point.

\begin{tabular}{lccc}
\hline Initial points & $S(6)$ & Thakur et al. (8) & $M(9)$ \\
\hline 2.2 & 23 & 13 & 8 \\
2.7 & 24 & 14 & 8 \\
3.3 & 25 & 14 & 8 \\
3.8 & 25 & 15 & 8 \\
4.3 & 26 & 15 & 8 \\
4.8 & 26 & 15 & 8 \\
\hline
\end{tabular}

$$
\begin{aligned}
|F a-F b| & =\left|2-\left(\frac{b-2}{2}\right)\right|=\left|\frac{b-2}{2}\right|=\frac{1}{3}\left(\left|\frac{3 b-6}{2}\right|\right) \\
& =\frac{1}{3}\left(\left|\frac{b-2}{2}+(b-2)\right|\right) \leq \frac{1}{3}\left|\frac{b-2}{2}\right|+\frac{1}{3}|b-2| \\
& =\frac{1}{3}\left|(b-a)+\left(a-\left(\frac{2+b}{2}\right)\right)\right|+\frac{1}{3}|b-2| \\
& \leq \frac{1}{3}|b-a|+\frac{1}{3}\left|a-\left(\frac{2+b}{2}\right)\right|+\frac{1}{3}|b-2| \\
& =\frac{1}{3}(|a-b|+|a-F b|+|b-F a|) .
\end{aligned}
$$

Next, for $a=4.2$ and $b=5,(1 / 2)|a-F a|<|a-b|$ but $|F a-F b|>|a-b|$. Hence, $F$ does not satisfy the $(C)$ condition. Let $\alpha_{n}=0.70, \beta_{n}=0.65$, and $\gamma_{n}=0.90$. The strong convergence of the $M(9)$, Thakur et al. (8), Abbas and Nazir (7), S (6), Noor (5), Ishikawa (4), and Mann (3) iterates to a fixed point $p=2$ is given in Table 1 .

Remark 12. From Table 1 and Figure 1, we see that the $M$ iteration process converges faster to $p=2$ than the others.

Now using the above example, we make different choices of parameters $\alpha_{n}$ and $\beta_{n}$ and intial points and also we get $\|$ $w_{n}-p \|<10^{-10}$ as our stopping criterion where $p=2$ is a 
TABLE 3: $\alpha_{n}=n /(n+5)^{10 / 9}$ and $\beta_{n}=1 /(n+5)^{2 / 3}$. Number of iterations required to obtain the fixed point.

\begin{tabular}{lccc}
\hline Initial points & $S(6)$ & Thakur et al. (8) & $M(9)$ \\
\hline 2.2 & 28 & 14 & 13 \\
2.7 & 29 & 15 & 14 \\
3.3 & 30 & 16 & 14 \\
3.8 & 31 & 16 & 14 \\
4.3 & 31 & 16 & 14 \\
4.8 & 31 & 16 & 14 \\
\hline
\end{tabular}

TABLE $4: \quad \alpha_{n}=((n+3) /(5 n+2))^{1 / 15}$ and $\beta_{n}=2 n /(5 n+100)^{1 / 4}$. Number of iterations required to obtain the fixed point.

\begin{tabular}{lccc}
\hline Initial points & $S(6)$ & Thakur et al. (8) & $M(9)$ \\
\hline 2.2 & - & 11 & 10 \\
2.7 & - & 12 & 11 \\
3.3 & - & 13 & 11 \\
3.8 & - & 14 & 11 \\
4.3 & - & 15 & 12 \\
4.8 & - & 15 & 12 \\
\hline
\end{tabular}

fixed point of $F$. The number of iterations for $M$ (9) to reach $p=2$ is compared with the leading three steps of Thakur et al. (8) and leading two steps of $S$ (6) iterations. The numbers in italic in Tables $2-4$ show that $M$ iteration is better than the others. The "-" represents that the number of iterations exceeds 50 .

\section{Data Availability}

No data were used to support this study.

\section{Conflicts of Interest}

The authors declare that they have no conflicts of interest.

\section{Authors' Contributions}

All authors contributed equally and significantly in writing this article. All authors read and approved the final manuscript.

\section{Acknowledgments}

The authors are grateful to the Spanish Government for Grant RTI2018-094336-B-I00 (MCIU/AEI/FEDER, UE) and to the Basque Government for Grant IT1207-19.

\section{References}

[1] T. Suzuki, "Fixed point theorems and convergence theorems for some generalized nonexpansive mappings," Journal of Mathematical Analysis and Applications, vol. 340, no. 2, pp. 1088-1095, 2008.
[2] A. Abkar and M. Eslamian, "A fixed point theorem for generalized nonexpansive multivalued mappings," Fixed Point Theory, vol. 12, no. 2, pp. 241-246, 2011.

[3] M. Basarir and A. Sahin, "On the strong and $\Delta$-convergence of $S$-iteration process for generalized nonexpansive mappings on CAT (0) spaces," Thai Journal of Mathematics, vol. 12, pp. 549-559, 2014.

[4] S. Dhompongsa, W. Inthakon, and A. Kaewkhao, "Edelstein's method and fixed point theorems for some generalized nonexpansive mappings," Journal of Mathematical Analysis and Applications, vol. 350, no. 1, pp. 12-17, 2009.

[5] B. Nanjaras, B. Panyanak, and W. Phuengrattana, "Fixed point theorems and convergence theorems for Suzuki generalized nonexpansive mappings in CAT (0) spaces," Nonlinear Analysis: Hybrid Systems, vol. 4, no. 1, pp. 25-31, 2010.

[6] W. Phuengrattana, "Approximating fixed points of Suzukigeneralized nonexpansive mappings," Nonlinear Analysis: Hybrid Systems, vol. 5, no. 3, pp. 583-590, 2011.

[7] B. S. Thakur, D. Thakur, and M. Postolache, "A new iterative scheme for numerical reckoning fixed points of Suzuki's generalized nonexpansive mappings," Applied Mathematics and Computation, vol. 275, pp. 147-155, 2016.

[8] I. Uddin and M. Imdad, "Convergence of SP-iteration for generalized nonexpansive mapping in Hadamard spaces," Hacettepe Journal of Mathematics and Statistics, vol. 47, no. 6, pp. 1595-1604, 2018.

[9] I. Uddin and M. Imdad, "On certain convergence of S-iteration scheme in CAT (0) spaces," Kuwait Journal of Science, vol. 42, no. 2, pp. 93-106, 2015.

[10] I. Uddin and M. Imdad, "Some convergence theorems for a hybrid pair of generalized nonexpansive mappings in CAT (0) spaces," Journal of Nonlinear and Convex Analysis, vol. 6, no. 3, pp. 447-457, 2015.

[11] K. Ullah and M. Arshad, "New iteration process and numerical reckoning xed point in Banach spaces," UPB Scientific Bulletin, Series A: Applied Mathematics and Physics, vol. 79, no. 4, pp. 113-122, 2017.

[12] K. Ullah and M. Arshad, "Numerical reckoning fixed points for Suzuki's generalized nonexpansive mappings via new iteration process," Univerzitet u Nišu, vol. 32, no. 1, pp. 187196, 2018.

[13] E. Karapinar, "Remarks on Suzuki (C)-condition," in Dynamical Systems and Methods, A. Luo, J. Machado, and D. Baleanu, Eds., pp. 227-243, Springer, New York, NY, 2012.

[14] K. Ullah, F. Ayaz, and J. Ahmad, "Some convergence results of $\mathrm{M}$ iterative process in Banach spaces," Asian-European Journal of Mathematics, vol. 2019, no. article 2150017, 2019.

[15] T. Abdeljawad, K. Ullah, and J. Ahmad, "On Picard-Krasnoselskii hybrid iteration process in Banach spaces," Journal of Mathematics, vol. 2020, Article ID 2150748, 5 pages, 2020.

[16] K. Ullah, J. Ahmad, and M. de la Sen, "On generalized nonexpansive maps in Banach spaces," Computation, vol. 8, no. 3, p. 61,2020 .

[17] T. Abdeljawad, K. Ullah, J. Ahmad, and N. Mlaiki, "Iterative approximations for a class of generalized nonexpansive operators in Banach spaces," Discrete Dynamics in Nature and Society, vol. 2020, Article ID 4627260, 6 pages, 2020.

[18] K. Ullah, M. S. U. Khan, N. Muhammad, and J. Ahmad, "Approximation of endpoints for multivalued nonexpansive mappings in geodesic spaces," Asian-European Journal of Mathematics, vol. 2019, no. article 2050141, 2019. 
[19] K. Ullah, J. Ahmad, and N. Muhammad, "Approximation of endpoints for multi-valued mappings in metric spaces," Journal of Linear and Toplogical Algebra, vol. 9, no. 2, pp. 129137, 2020.

[20] K. Ullah, J. Ahmad, M. de la Sen, and M. N. Khan, “Approximating fixed points of Reich-Suzuki type nonexpansive mappings in hyperbolic spaces," Journal of Mathematics, vol. 2020, Article ID 2169652, 6 pages, 2020.

[21] W. R. Mann, "Mean value methods in iteration," Proceedings of American Mathematical Society, vol. 4, no. 3, pp. 506-510, 1953.

[22] S. Ishikawa, "Fixed points by a new iteration method," Proceedings of American Mathematical Society, vol. 44, no. 1, pp. 147-150, 1974.

[23] M. A. Noor, "New approximation schemes for general variational inequalities," Journal of Mathematical Analysis and Applications, vol. 251, no. 1, pp. 217-229, 2000.

[24] R. P. Agarwal, D. O'Regon, and D. R. Sahu, "Iterative construction of fixed points of nearly asymtotically nonexpansive mappings," Journal of Nonlinear and Convex Analysis, vol. 8, no. 1, pp. 61-79, 2007.

[25] M. Abbas and T. Nazir, "A new faster iteration process applied to constrained minimization and feasibility problems," Math Vesnik, vol. 66, no. 2, pp. 223-234, 2014.

[26] Z. Opial, "Weak convergence of the sequence of successive approximations for nonexpansive mappings," Bulletin of the American Mathematical Society, vol. 73, no. 4, pp. 591-598, 1967.

[27] H. F. Senter and W. G. Dotson, "Approximating fixed points of nonexpansive mappings," Proceedings of the American Mathematical Society, vol. 44, no. 2, pp. 375-380, 1974.

[28] J. A. Clarkson, "Uniformly convex spaces," Transactions of the American Mathematical Society, vol. 40, no. 3, pp. 396-414, 1936.

[29] R. P. Agarwal, D. O'Regan, and D. R. Sahu, Fixed Point Theory for Lipschitzian-Type Mappings with Applications Series, Topological Fixed Point Theory and Its Applications, vol. 6, Springer, New York, 2009.

[30] W. Takahashi, Nonlinear Functional Analysis, Yokohoma Publishers, Yokohoma, 2000.

[31] J. Schu, "Weak and strong convergence to fixed points of asymptotically nonexpansive mappings," Bulletin of the Australian Mathematical Society, vol. 43, no. 1, pp. 153-159, 1991. 